\title{
Erratum to: Engineering of Landau-Zener tunneling
}

\author{
G. Tayebirad $\cdot$ R. Mannella $\cdot$ S. Wimberger
}

Published online: 10 January 2015

(C) Springer-Verlag Berlin Heidelberg 2015

\section{Erratum to: Appl Phys B (2011) 102:489-495 DOI 10.1007/s00340-011-4430-6}

In the original publication of the article, the inset of Fig. 2 was published incorrectly. The correct inset of Fig. 2 is reported here.

The tunneling of an initial quantum wave packet prepared in the ground band of a periodic lattice was studied in Ref. [1] for the first time in a time-resolved manner. If the initial wave packet is of a size in momentum space much smaller than the Brillouin zone of the lattice, the survival probability of this packet when accelerated toward the Brillouin zone shows a characteristic step-like structure [1]. The details of the survival probability were analyzed in more detail in Refs. [2,3]. The widths of the steps were studied as a function of the extension of the initial state in momentum space in Fig. 2 of Ref. [2]. In this figure, the data shown in the inset are not correct. The inset should be substituted by Fig. 1 below. Details on the shown quantities and units are found in $[2,3]$.

The online version of the original article can be found under doi:10.1007/s00340-011-4430-6.

\section{G. Tayebirad $(\bowtie) \cdot S$. Wimberger}

Institut für Theoretische Physik, Universität Heidelberg,

Philosophenweg 19, 69120 Heidelberg, Germany

e-mail: s.wimberger@thphys.uni-heidelberg.de

R. Mannella

CNR-INFM and Dipartimento di Fisica 'E. Fermi',

Università di Pisa, Largo Pontecorvo 3, 56127 Pisa, Italy

S. Wimberger

Center for Quantum Dynamics, Universität Heidelberg,

Heidelberg, Germany

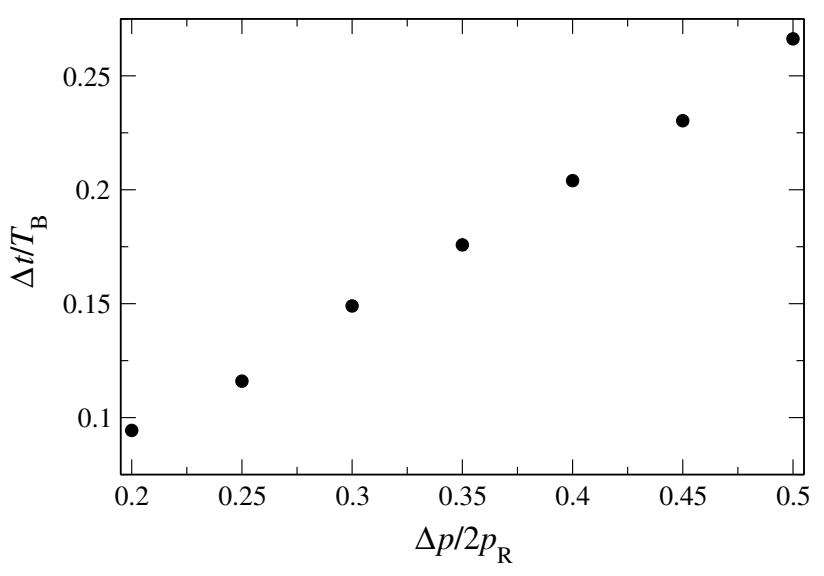

Fig. 1 Step width $\Delta t$ (of the survival probability vs. time) in units of the Bloch time $T_{B}$ as a function of the extension of the initial state in momentum space $\Delta p$ in units of 2-photon-recoils $2 p_{\mathrm{R}}$ (filled circles). The observed linear relationship means that the wave packet needs the more time to tunnel the wider it is in momentum space, while it is scanned over the band edge of the Brillouin zone where the tunneling takes place

\section{References}

1. A. Zenesini, H. Lignier, G. Tayebirad, J. Radogostowicz, D. Ciampini, R. Mannella, S. Wimberger, O. Morsch, E. Arimondo, Phys. Rev. Lett. 103, 090403 (2009)

2. G. Tayebirad, R. Mannella, S. Wimberger, Appl. Phys. B 102, 489 (2011)

3. G. Tayebirad, R. Mannella, S. Wimberger, Phys. Rev. A 84, 031605(R) (2011) 